
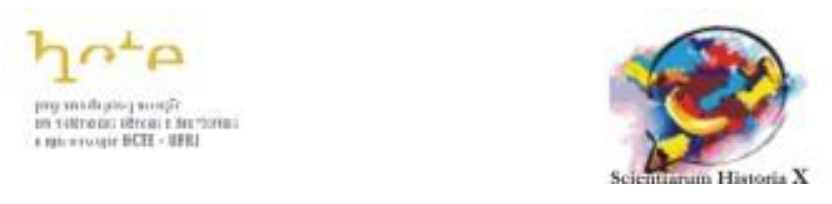

\title{
A Matemática através da resolução de problemas: uma experiência na Seeduc-RJ
}

\section{Mathematics through problem solving: an experience at Seeduc-RJ}

\author{
Darlan Azevedo Gomes \\ Secretaria de Estado de Educação do Rio de Janeiro (Seeduc-RJ) \\ profdag@gmail.com \\ orcid.org/0000-0001-7108-4932
}

\section{Augusto Cesar de Castro Barbosa}

Departamento de Matemática Aplicada, Instituto de Matemática e Estatística, Universidade do Estado do Rio de Janeiro (UERJ)

accb@ime.uerj.br

orcid.org/0000-0002-5094-1509

\section{Cláudia Ferreira Reis Concordido}

Departamento de Análise Matemática, Instituto de Matemática e Estatística,

Universidade do Estado do Rio de Janeiro (UERJ)

concordido@ime.uerj.br

orcid.org/0000-0002-0767-9170

\begin{abstract}
Resumo. O objetivo deste trabalho é abordar algumas concepções acerca da Resolução de Problemas, em especial, no âmbito da Secretaria de Estado de Educação do Rio de Janeiro (Seeduc). Analisamos a disciplina Resolução de Problemas Matemáticos (RPM), lançada pela Seeduc em 2012, apresentando a visão da Secretaria e a de um grupo de professores pertencentes à rede estadual de ensino. Para isso, foi discutido o documento da secretaria que norteia a implantação da disciplina RPM e foi realizada uma breve pesquisa de perfil e opinião com esse grupo de professores.
\end{abstract}

Palavras-chave: Ensino de Matemática. Resolução de Problemas. Secretaria de Estado de Educação do Rio de Janeiro.

Abstract. The purpose of this work is to approach some conceptions about Problem Solving, especially within the scope of the Rio de Janeiro State Department of Education (Seeduc). We analyzed the discipline Mathematical Problem Solving 
(RPM), created by Seeduc in 2012, presenting the vision of the Secretariat and that of a group of teachers belonging to the state school system. For this, the secretary's document that guides the implementation of the RPM discipline was discussed and a brief profile and opinion survey was conducted with this group of teachers.

Keywords:Math Teaching. Problem Solving. Rio de Janeiro State Department of Education.

Recebido: 01/10/2017 Aceito: 27/10/10 Publicado: 05/11/2017

\section{Introdução}

Um ponto importante relativo ao baixo desempenho escolar, em especial em Matemática, é a dificuldade em lidar com situações que exijam um raciocínio lógico aprimorado. Nesse sentido, é papel do professor propiciar condições para o desenvolvimento do raciocínio lógico e da capacidade de abstração e análise, em suma, o de fazer pensar.

Uma das maneiras de proporcionar essas condições é a utilização da resolução de problemas como metodologia de ensino, pois ela é capaz de criar mecanismos que propiciam aos alunos um ambiente de descobertas. De acordo com os Parâmetros Curriculares Nacionais (PCN) - Matemática (BRASIL, 1997, p. 32), no "processo de ensino e aprendizagem, conceitos, ideias e métodos matemáticos devem ser abordados mediante a exploração de problemas, ou seja, de situações em que os alunos precisem desenvolver algum tipo de estratégia para resolvê-las".

Atenta ao fato de que a resolução de problemas é considerada como um recurso ao ensino da Matemática e tendo por objetivo melhorar o desempenho nas habilidades cognitivas relacionadas à resolução de problemas, Seeduc criou no ano de 2012, através da Resolução n 4843 (RIO DE JANEIRO, 2012), a disciplina Resolução de Problemas Matemáticos (RPM). Essa é disciplina à parte da Matemática e começou a ser lecionada já no início do ano letivo de 2013.

Após algum tempo de existência da disciplina RPM, era de se esperar que as atividades desenvolvidas pelos professores em sala de aula já estivessem em consonância com as orientações dadas pela Seeduc. Assim, o nosso objetivo com esse trabalho foi verificar se a disciplina RPM vinha cumprindo as metas traçadas pela Seeduc.

Dessa forma, foram analisados os documentos da Seeduc que tratam da RPM e foi aplicado um questionário a um grupo de professores que lecionam em escolas situadas em Campo Grande, bairro da Zona Oeste do município do Rio de Janeiro. A partir das informações obtidas desse questionário, conseguiu-se um indicativo do que pensam e de 
como trabalham a disciplina RPM alguns professores da rede estadual do Rio de Janeiro.

\section{A Resolução de Problemas em Matemática}

Segundo Polya (1995), uma pessoa está diante de um problema quando ela se depara com uma questão que não pode responder ou resolver usando os seus conhecimentos. Dante (1991) afirma que problema "é qualquer situação que exija o pensar do indivíduo para solucioná-la". Segundo Van de Walle (2001, apud ONUCHIC, ALLEVATO, 2005), “[...] um problema é definido como qualquer tarefa ou atividade para a qual os estudantes não têm métodos ou regras prescritas ou memorizadas, nem a percepção de que haja um método específico para chegar à solução correta". Onuchic (1999) afirma que um problema pode ser caracterizado como sendo tudo aquilo que não se sabe fazer, porém se está interessado em resolver.

A escolha do problema a ser trabalhado desempenha um papel de fundamental importância, na medida em que é necessário despertar no aluno não apenas o interesse pelo assunto envolvido no problema, mas também o interesse na busca de sua solução. Dessa forma, nos resta pensar de que maneira podemos fazer uso de problemas em sala de aula para desenvolver habilidades e competências matemáticas em nossos alunos.

Polya (1995) sugere quatro etapas principais para a resolução de um problema. A primeira consiste em compreender o problema. Nesta etapa se elaboram perguntas a fim de descobrir o que é solicitado: quais os dados e as condições, que algoritmos podem ser utilizados, etc. A segunda etapa é a construção de uma estratégia de resolução, que demanda que se estabeleçam relações entre os dados do problema e o que nele se pede, que se busquem algoritmos ou mesmo problemas parecidos já resolvidos. Na terceira etapa, em que ocorre a execução da estratégia, deve-se verificar cada passo dado e efetuar as operações necessárias para se chegar ao resultado esperado. Finalmente, na quarta etapa faz-se o retrospecto ou verificação do resultado. Nesse ponto avalia-se o limite de validade da solução encontrada e também a possibilidade de se chegar ao resultado por um caminho diferente.

Para Dante (1991), a resolução de problemas não deve constituir-se de experiências repetitivas, cabendo ao professor sugerir diferentes maneiras de solucioná-los, destacando sempre que não há uma forma única, ideal e infalível para alcançar a resolução. É necessário que o aluno perceba que pode solucionar diferentes problemas utilizando a mesma estratégia, bem como pode utilizar estratégias diversas para a solução de um único problema.

Mendonça (1993, p. 260) destaca três dimensões para a resolução de problemas: ensinar sobre resolução de problemas, ensinar para a resolução de problemas e ensinar através da resolução de problemas. Na primeira concepção, a resolução de problemas é vista 
como um processo, como um meio para desenvolver o potencial heurístico do aluno. A segunda tem a resolução de problemas como um objetivo, como uma meta final. $\mathrm{Na}$ terceira, a resolução de problemas é um ponto de partida, que dispara um processo de construção do conhecimento matemático. Acreditamos que a terceira concepção, em que o conteúdo matemático seja introduzido por uma situação-problema, permite que fique mais clara a necessidade de se construir os conceitos matemáticos desejados.

A resolução de problemas é uma metodologia que dá a oportunidade aos estudantes de buscar uma solução para o problema proposto, de forma que eles são levados a exercitar as suas habilidades intelectuais. Desse modo, a resolução de problemas estreita a distância entre uma Matemática mais intuitiva, baseada na experiência do dia a dia e a Matemática formal.

No entanto, para Onuchic e Allevato (2011), trabalhar com a resolução de problemas exige que professores e alunos tenham novas posturas e atitudes com relação ao trabalho desenvolvido em sala de aula. O professor deve escolher os problemas adequados ao conceito que vai ser construído. Por outro lado, os alunos passam a ter maior participação e responsabilidade no processo de aprendizagem.

Dante (2011, p. 14) afirma que "ensinar a resolver problemas não é um mecanismo direto de ensino, mas uma variedade de processos de pensamento que precisam ser cuidadosamente desenvolvidos pelo aluno com o apoio e incentivo do professor". Ele afirma também que "é preciso desenvolver no aluno a habilidade de elaborar raciocínios lógicos e fazer uso inteligente e eficaz dos recursos disponíveis, para que ele possa propor boas soluções às questões que surgem em seu dia a dia, na escola ou fora dela". Para o autor, diversas estratégias para a resolução de um problema devem ser propostas às crianças, mostrando que não existe uma única estratégia correta e infalível e que, além disso, cada problema pode exigir uma específica (Dante, 2011).

\section{A disciplina Resolução de Problemas Matemáticos}

O principal objetivo da disciplina RPM, na visão da Seeduc, é o desenvolvimento da capacidade dos alunos em resolver situações-problema relacionadas ao seu nível escolar, de modo que o raciocínio matemático desenvolvido auxilie o professor da disciplina do curso regular na abordagem dos conteúdos. A RPM não deve representar uma ampliação da carga horária da disciplina Matemática, mas ser uma disciplina independente, com planejamento próprio e que não introduza conceitos e sim os retome.

Desde 2013 a disciplina RPM vem sendo oferecida do $6^{\circ}$ ao $9^{\circ}$ ano do Ensino Fundamental e no $2^{\circ}$ ano do Ensino Médio. Ela também visa desenvolver habilidades e competências que contribuam às demais disciplinas, possibilitando assim a formação de um cidadão em condições plenas de lidar, em seu cotidiano, com situações em que o raciocínio lógico esteja presente. 
O documento elaborado pela Seeduc leva em consideração a análise de diferentes abordagens para a resolução de problemas, o Currículo Mínimo de Matemática do Ensino Regular e as habilidades e competências relativas à Matemática que são fundamentais para outras disciplinas.

A fim de verificar o conhecimento e visão dos professores sobre os objetivos e habilidades da disciplina RPM determinados pela Seeduc, aplicamos um questionário para um grupo de 40 professores de Matemática lotados em escolas estaduais no entorno do bairro de Campo Grande, Rio de Janeiro - RJ.O questionário foi composto de questões relativas ao perfil profissional e à prática docente desse grupo de professores. Esse levantamento mostrou que a maioria dos professores possui idade entre 40 e 49 anos e que terminou a graduação entre 2000 e 2003, com uma média de 8,5 anos de prática docente. Em relação à formação acadêmica, a mais alta titulação obtida por esse grupo é a de especialização. Percebe-se nas respostas a importância dada à continuidade dos estudos, pois quase $70 \%$ possuem algum curso de pós-graduação.

Quanto à carga horária semanal de trabalho, obtivemos a seguinte distribuição: 13\% lecionavam mais de $40 \mathrm{~h}, 20 \%$ de $30 \mathrm{~h}$ a $40 \mathrm{~h}, 13 \%$ de $20 \mathrm{~h}$ a $30 \mathrm{~h}, 20 \%$ de $12 \mathrm{~h}$ a $20 \mathrm{~h}$ e $34 \%$ até $12 \mathrm{~h}$. Quanto às horas semanais dedicadas ao planejamento das atividades de sala de aula, os resultados obtidos foram: $13 \%$ dedicam mais de $8 \mathrm{~h}, 20 \%$ de $4 \mathrm{~h}$ a $8 \mathrm{~h}$, $60 \%$ até $4 \mathrm{~h}$ e $7 \%$ não planejam. Quanto à prática docente, 21 professores já tinham lecionado ou estavam lecionando a disciplina RPM. No entanto, 24 deles a consideraram uma ampliação da carga horária de Matemática - o que contraria as orientações da Seeduc.

Foi solicitado que os professores indicassem qual seria o principal objetivo da disciplina RPM definido pela Seeduc, dentre vários objetivos elencados. Uma grande parcela conhecia o objetivo principal da disciplina. Isso pode ser considerado um aliado ao desenvolvimento da prática docente, pois conhecer os objetivos proporciona maior segurança ao professor quanto à escolha dos meios mais apropriados para atingi-los. Os resultados dessa questão aparecem na Figura 1. 

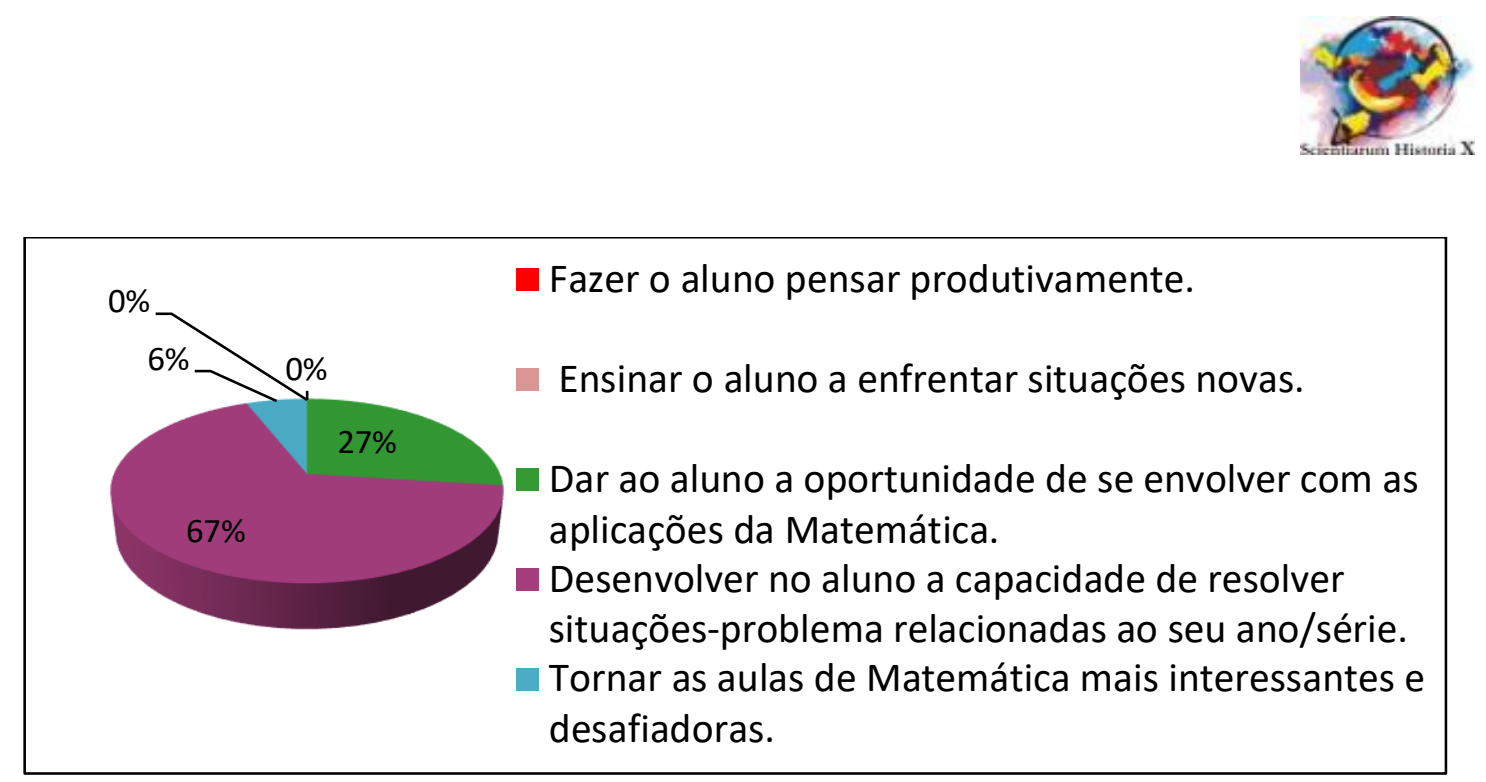

Figura 1. Gráfico com a descrição dos principais objetivos definidos pela Seeduc para a disciplina RPM.

Fonte: Autores.

A pergunta seguinte objetivou descobrir quais vantagens as atividades desenvolvidas durante as aulas de RPM trariam aos alunos (Figura 2). Uma das respostas com maior percentual afirma que as atividades têm possibilitado aos alunos gravar as regras que permitem obter respostas certas dos cálculos e problemas, porém essa afirmação não se enquadra na metodologia da resolução de problemas nem tampouco nos objetivos propostos pela Seeduc para a disciplina. Esse fato vai de encontro à resposta dada à questão representada pelo gráfico da Figura 1. Cabe destacar que apenas neste questionamento mais de um item poderia ser sinalizado.

Foi destinado também um espaço para que os professores pudessem opinar sobre as abordagens que a disciplina RPM poderia ter no sentido de aprimorá-la. Dentre as respostas obtidas, podemos destacar: uma abordagem mais contextualizada com a realidade do aluno, envolvimento da Matemática em projetos interdisciplinares, necessidade de maior autonomia do professor para trabalhar outros temas além dos instituídos pela Seeduc, trabalhar conteúdos presentes em provas de concursos e inserção da RPM no laboratório de Matemática.

Ficou evidenciado ainda que alguns dos professores desconhecem as orientações curriculares fornecidas pela Seeduc, apesar de a maioria indicar corretamente o principal objetivo nela descrito e de o início da disciplina RPM ter ocorrido no começo do ano letivo de 2013. É importante destacar que em momento algum a Seeduc capacitou ou criou meios para que os professores pudessem tirar dúvidas, trocar experiências ou conhecer essa disciplina. 

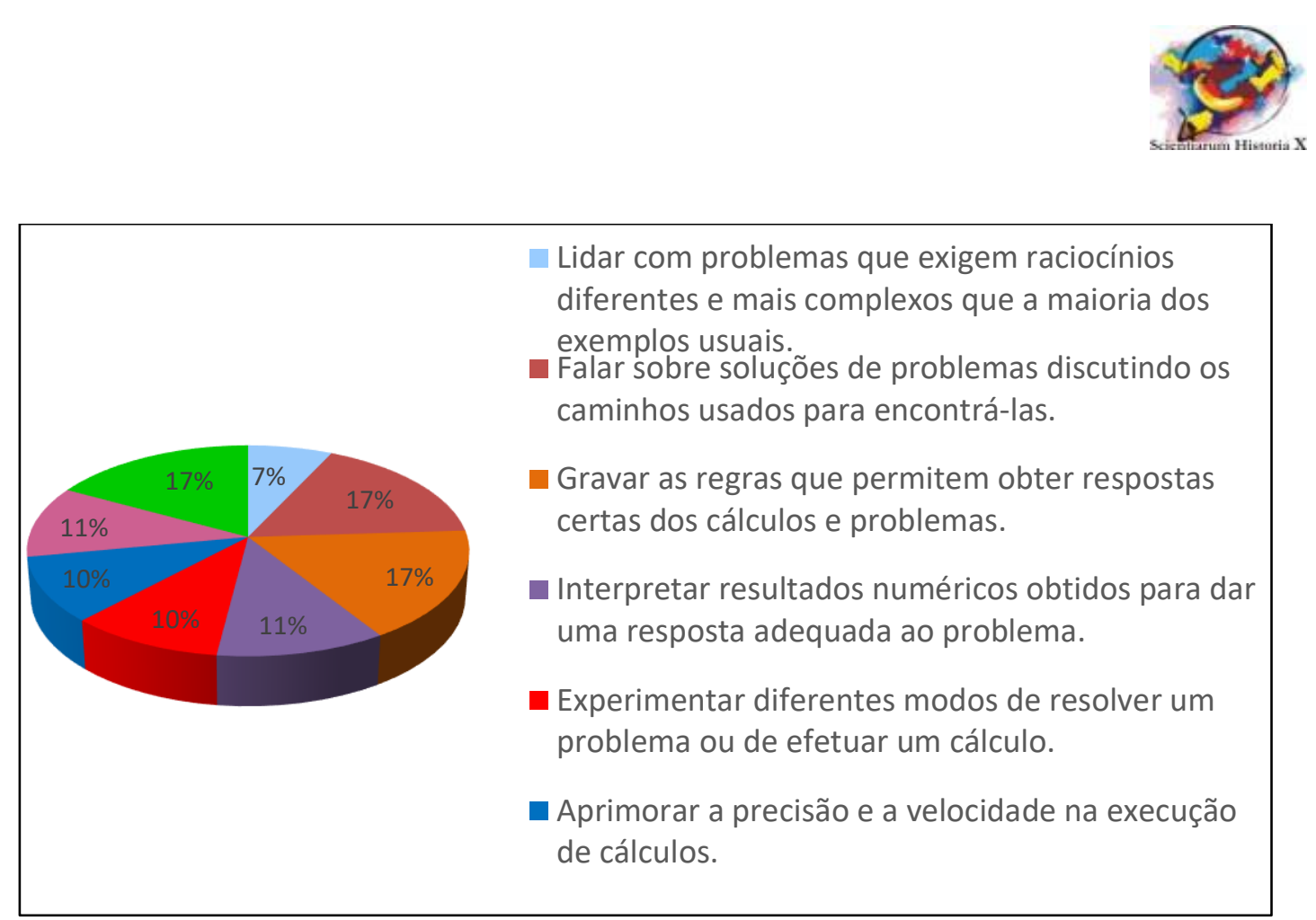

Figura 2. Objetivos alcançados a partir da disciplina RPM.

Fonte: Autores.

Vale ressaltar que a matriz curricular publicada no Diário Oficial do Estado do Rio de Janeiro, do dia 11 de maio de 2016, não trouxe a obrigatoriedade da oferta da disciplina RPM para o ano letivo de 2017. Apesar disso, essa disciplina continua a ser oferecida em algumas escolas e faz parte das disciplinas regulares do currículo mínimo do Ensino Fundamental (anos finais) e Médio no site da Seeduc.

\section{Conclusões}

Ao implantar a disciplina RPM, entendemos que a Seeduc esperava tornar os alunos cidadãos aptos a encarar as diversas situações do dia a dia nas quais o raciocínio matemático fosse fundamental. A Secretaria definiu ainda um currículo mínimo, integrado ao currículo de Matemática, para orientar os professores de RPM quanto aos objetivos a serem alcançados.

A partir das informações coletadas do questionário aplicado ao grupo de 40 professores, verificou-se que 37 deles dedicam algum tempo ao planejamento de suas aulas e, portanto, eles deveriam saber quais são esses objetivos. Porém, para 13 desses professores, gravar as regras que permitem obter respostas certas dos cálculos e problemas é um dos objetivos a serem alcançados, o que evidencia desconhecimento das orientações da Seeduc sobre a disciplina.

É possível afirmar que a resolução de problemas, seja na forma de uma disciplina específica, como a proposta pela Seeduc, seja como metodologia adotada nas aulas 
regulares de Matemática, é uma ferramenta útil no processo de construção do conhecimento. Assim, para o seu correto desenvolvimento, é necessário que o professor dedique parte do seu tempo ao planejamento/seleção de atividades que estimulem a curiosidade, a intuição, o interesse em buscar diversas soluções para o problema apresentado, bem como atender ao conteúdo programático estabelecido.

Ao nos depararmos com a não obrigatoriedade da disciplina RPM, surge a seguinte pergunta: e agora? A fim de que não se percam nem tenham sido em vão as propostas definidas pela Seeduc à RPM, seria salutar adotar as seguintes medidas: inserção da metodologia de resolução de problemas nas aulas de Matemática a partir do sexto ano do Ensino Fundamental; oferecimento, num ambiente virtual de aprendizagem, de capacitações sobre as perspectivas da resolução de problemas; criação de um canal eficaz de comunicação entre os diversos professores de Matemática pertencentes aos quadros da Seeduc com grupos de pesquisas já estabelecidos na área de resolução de problemas; realização de seminários sobre o tema em questão.

\section{Referências}

BRASIL. Secretaria de Educação Fundamental. Parâmetros Curriculares Nacionais: Matemática / Secretaria de Educação Fundamental. Brasília: MEC / SEF, 1997.

BRASIL. Secretaria da Educação Média e Tecnológica. PCN+ Ensino Médio:Orientações Curriculares Complementares aos Parâmetros Curriculares Nacionais. Ciências da Natureza, Matemática e suas Tecnologias. Brasília: Ministério da Educação, 2002.

DANTE, L. R. Didática da resolução de problemas de Matemática. 2. ed. São Paulo: Ática, 1991.

DANTE, L. R. Formulação e resolução de problemas de matemática: teoria e prática. São Paulo: Ática, 2011.

MENDONÇA, M. C. D. Problematização: Um caminho a ser percorrido em Educação Matemática. 1993. 307 f. Tese (Doutorado em Educação) - Faculdade de Educação, Universidade Estadual de Campinas, Campinas, 1993.

OCDE. Organização de cooperação e de desenvolvimento econômico. Programme for International Student Assessment (PISA). Resultsfrom PISA 2015. 2016. Disponível em: <http://www.oecd.org/pisa/PISA-2015-Brazil-PRT.pdf>. Acesso em: 02 mar. 2017. 
ONUCHIC, L. R. Ensino-Aprendizagem de Matemática através da Resolução de Problemas. In: BICUDO, M. A. V (Org.). Pesquisa em Educação Matemática: Concepções e Perspectivas. São Paulo: Ed. UNESP, 1999.

ONUCHIC, L. R.; ALLEVATO, N. S. G. Novas reflexões sobre o ensino-aprendizagem através da Resolução de Problemas. In: BICUDO, M. A. V.; BORBA, M. C.(Orgs.). Educação matemática: pesquisa em movimento. São Paulo: Cortez, 2005.

ONUCHIC, L. R.; ALlEVATO, N. S. G. Pesquisa em Resolução de Problemas: caminhos, avanços e novas perspectivas. Boletim de educação Matemática, Rio Claro, SP, v. 25, n. 41, p. 73-98, dez. 2011. Disponível em: <http://www.redalyc.org/articulo.oa?id=291223514005>.

Acesso em: 29 abr. 2015.

POLYA, G. A arte de resolver problemas: um novo aspecto do método matemático. Tradução e adaptação de Heitor Lisboa de Araújo. 2. reimpr. Rio de Janeiro: Interciências, 1995.

RIO DE JANEIRO. Secretaria de Estado de Educação. Resolução Seeduc N 4843 de 03.12.2012 - Diretrizes Implantação Matrizes Curric. Educação Basica. Rio de Janeiro: Seeduc, 2012. 